\title{
ATP and cough reflex hypersensitivity: a confusion of goals?
}

\author{
To the Editor:
}

The term "cough hypersensitivity syndrome" (CHS) has been proposed by a European Respiratory Society Task Force as a clinical syndrome characterised by troublesome coughing often triggered by low levels of thermal, mechanical or chemical exposure, in patients presenting with chronic cough [1]. There are no agreed clinical criteria defining this syndrome or diagnostic tests to confirm its presence. However, it is often assumed that this syndrome must be linked to a hypersensitivity of the vagal afferent nerves and an intrinsic defect in the nerves themselves, which may not be the case [2]. The paper by FowLes et al. [3] describes a tussive challenge study with ATP and AMP in healthy volunteers and unselected patients attending a cough clinic, demonstrating the greater potency of ATP versus AMP, and that chronic cough patients had a greater cough response at lower concentrations of ATP. The degree of hypersensitivity demonstrated in chronic cough patients did not appear to be greater in magnitude than seen previously with capsaicin challenge (the only other example where an intervention against a specific challenge agent has been tested) [4-6]. However, a TRPV1 receptor antagonist did not impact on cough in this patient group despite a greater sensitivity to capsaicin challenge [6]. The authors have concluded, therefore, that 1) a similar shift in sensitivity in the ATP challenge does not explain the efficacy of the P2X 3 antagonist (AF-219/MK-7264) and that 2) it is not a peripheral response to ATP that underlies the increased coughing in these patients.

This is an important and interesting study, but we would not agree with the conclusions proffered by the authors or in the accompanying commentary [7] and would suggest that the shift in response cannot be compared to any other tussive agents. Firstly, it is notable that this study did not investigate well-phenotyped patients with refractory (idiopathic) chronic cough, the specific group shown to be highly responsive to P2X3 antagonism [8]. Furthermore, whether a shift in the coughing to ATP challenge predicts clinical efficacy of AF-219/MK-7264 can only be established when the cough challenge and the compound are tested in follow-up studies in refractory chronic cough, unselected chronic cough and other patient groups that exhibit chronic cough. If efficacy is established in more than one group with a similar shift in the ATP challenge response, then an ATP challenge may well predict clinical efficacy of AF-219/MK-7264 or other P2X3 antagonists.

Secondly, even if the sensitivity of the nerve to exogenous challenge agents does not turn out to predict clinical efficacy, this does not rule out that it is a peripheral response to ATP that underlies the enhanced cough in these patients. Increased coughing may be due to 1) phenotypic change of the nerve and increased expression of the relevant target (e.g. P2X3 ion channel), 2) peripheral or central sensitisation of the nerve so that it responds at lower thresholds (the hypothesis suggested by the authors), or 3) elevated levels of the endogenous activating mediator produced by the diseased lung (e.g. ATP). In the latter case, there would be no requirement for an increase in the nerve sensitivity to exogenous agents. We suggest that at least in patients with chronic idiopathic cough that respond to AF-219/MK-7264, this may be the case. Our hypothesis is based on two pieces of evidence: 1) AF-219/MK-7264 is a peripherally restricted drug [8] and 2) capsaicin challenge in refractory chronic cough patients is not blocked by AF-219/ MK-7264 [9], suggesting that the enhanced responses to other tussive agents are not necessarily driven by ATP. All this evidence points to the conclusion that enhanced cough in patients who are treatment sensitive is due to increased levels of ATP produced in the lung, perhaps by upregulation of ATP releasing mechanisms such as the TRPV4/pannexin axis [10], providing an increased sensory afferent drive to evoke cough. We would suggest that the term "CHS" is used only to describe the exaggerated cough seen in patients, rather than confused with the terminology used to describe the underlying mechanisms.

@ERSpublications

The term "cough hypersensitivity syndrome" describes exaggerated cough but not necessarily the mechanisms involved http://ow.ly/Wk0n30bWmAy

Cite this article as: Belvisi MG, Smith JA. ATP and cough reflex hypersensitivity: a confusion of goals? Eur Respir J 2017; 50: 1700579 [https://doi.org/10.1183/13993003.00579-2017]. 
Maria G. Belvisi ${ }^{1}$ and Jaclyn A. Smith ${ }^{2}$

${ }^{1}$ Respiratory Pharmacology Group, Division of Airway Disease, National Heart and Lung Institute, Imperial College London, London, UK. ${ }^{2}$ Division of Infection, Immunity and Respiratory Medicine, University of Manchester, University Hospital of South Manchester, Manchester Academic Health Sciences Centre, Manchester, UK.

Correspondence: Maria G. Belvisi, Respiratory Pharmacology Group, Pharmacology and Toxicology Section, NHLI 1st Floor, Room 109, Sir Alexander Fleming Building, South Kensington Campus, Exhibition Road, Imperial College London, London SW7 2AZ, UK. E-mail: m.belvisi@imperial.ac.uk

Received: March 202017 | Accepted after revision: April 042017

Conflict of interest: Disclosures can be found alongside this article at erj.ersjournals.com

\section{References}

1 Morice AH, Millqvist E, Belvisi MG, et al. Expert opinion on the cough hypersensitivity syndrome in respiratory medicine. Eur Respir J 2014; 44: 1132-1148.

2 Bonvini SJ, Birrell MA, Smith JA, et al. Targeting TRP channels for chronic cough: from bench to bedside. Naunyn Schmiedebergs Arch Pharmacol 2015; 388: 401-420.

3 Fowles HE, Rowland T, Wright C, et al. Tussive challenge with ATP and AMP: does it reveal cough hypersensitivity? Eur Respir J 2017; 49: 1601452.

4 Prudon B, Birring SS, Vara DD, et al. Cough and glottic-stop reflex sensitivity in health and disease. Chest 2005; 127: 550-557.

5 Hilton EC, Baverel PG, Woodcock A, et al. Pharmacodynamic modeling of cough responses to capsaicin inhalation calls into question the utility of the C5 end point. J Allergy Clin Immunol 2013; 132: 847-855.

6 Khalid S, Murdoch R, Newlands A, et al. Transient receptor potential vanilloid 1 (TRPV1) antagonism in patients with refractory chronic cough: a double-blind randomized controlled trial. J Allergy Clin Immunol 2014; 134: 56-62.

$7 \quad$ Birring SS. The search for the hypersensitivity in chronic cough. Eur Respir J 2017; 49: 1700082.

8 Abdulqawi R, Dockry R, Holt K, et al. P2X3 receptor antagonist (AF-219) in refractory chronic cough: a randomised, double-blind, placebo-controlled phase 2 study. Lancet 2015; 385: 1198-1205.

9 Smith JA, Kitt M, Butera P, et al. The effect of P2X3 antagonism (AF-219) on experimentally evoked cough in healthy volunteers and chronic cough patients. Thorax 2016; 71: Suppl. 3, A18-A19.

10 Bonvini SJ, Birrell MA, Grace MS, et al. Transient receptor potential cation channel, subfamily V, member 4 and airway sensory afferent activation: Role of adenosine triphosphate. J Allergy Clin Immunol 2016; 138: 249-261 e212.

Copyright @ERS 2017

From the authors:

We thank our friends M.G. Belvisi and J.A. Smith for their interest in our article. We are in agreement that cough hypersensitivity syndrome (CHS) is a clinical diagnosis, currently without a firm pathophysiological explanation. The exquisite sensitivity of patients with CHS to a wide variety of irritants is attested in every clinic and is a cardinal feature of the clinical history of this condition. The stunning effectiveness of AF-219/MK-7264 in chronic cough led us to the assumption that ATP inhalation may well be the physiological tool to define CHS just as methacholine hyperresponsiveness is useful in defining the phenomenon of bronchial hyperresponsiveness. Sadly, our results did not confirm this expectation. Our patients were not "uncharacterised" but had been through our well-established clinic protocol. They were entirely typical of chronic cough patients. The failure of ATP challenge to demonstrate a marked leftward shift in the concentration response curve in chronic cough is puzzling, but we have difficulty understanding M.G. Belvisi and J.A. Smith's alternative explanation. AF-219/MK-7264 is thought to have a mainly peripheral activity; however, the periphery will also include the nodose and jugular ganglia of the vagus nerve, where phenotypic switching has been well described [1]. Our observation that hypersensitivity in chronic cough was no more demonstrated by ATP than citric acid or capsaicin does, to us, suggest that this phenomenon resides not on the nerve terminals where we had for many years assumed it to be, but rather be part of a pan-neuronal phenomenon.

Much of the conjecture raised by M.G. Belvisi and J.A. Smith will be answered by the analysis of as yet unpublished studies of AF-219/MK-7264. In our hands, ATP challenge was significantly ameliorated by AF-219/MK-7264, demonstrating target engagement in the periphery. Interestingly, whilst there was little

@ERSpublications

Cough hypersensitivity is a mystery that continues to require unravelling http://ow.ly/Fhel $30 \mathrm{cgA} 5 \mathrm{u}$

Cite this article as: Fowles HE, Rowland T, Wright C, et al. ATP and cough reflex hypersensitivity: a confusion of goals? Eur Respir J 2017; 50: 1700802 [https://doi.org/10.1183/13993003.00802-2017]. 
effect on citric acid or capsaicin inhalation, distilled water challenge was significantly altered, supporting their elegant hypothesis that the osmotically sensitive TRPV4/pannexin pathway may be a major effector mechanism in the CHS. That exogenous ATP does not stimulate excessive cough response suggests ATP is not the hypersensitiser. The fact that we have not yet unravelled the mechanism of CHS does not nullify the patient's observation of the phenomenon.

Helen Elizabeth Fowles ${ }^{1}$, Tim Rowland ${ }^{2}$, Caroline Wright ${ }^{1}$ and Alyn Morice ${ }^{1}$

${ }^{1}$ Hull York Medical School Centre for Cardiovascular and Metabolic Research, Respiratory, Castle Hill Hospital, Cottingham, UK. ${ }^{2}$ Castle Hill Hospital, Cottingham, UK.

Correspondence: Helen Elizabeth Fowles, Hull York Medical School Centre for Cardiovascular and Metabolic Research, Respiratory, Castle Hill Hospital, Cottingham, HU16 5JQ, UK. E-mail: FowlesHE@cardiff.ac.uk

Received: April 172017 | Accepted after revision: April 252017

Conflict of interest: Disclosures can be found alongside this article at erj.ersjournals.com

\section{Reference}

1 Mazzone SB, Undem BJ. Vagal afferent innervation of the airways in health and disease. Physiol Rev 2016; 96: 975-1024. 